\title{
UNA APROXIMACIÓN TEÓRICA A LA PLANEACIÓN EN LAS ORGANIZACIONES
}

\author{
A theORETICAL APPROACH TO PLANNING IN ORganizations \\ Desiderio López Niño* \\ Yeimi Jimena Cárdenas Santamaría**
}

Recibido: 18 de julio de 2017

Aceptado: 14 de noviembre de 2017

\section{Resumen}

Este artículo surge dentro del marco del proyecto de investigación sobre el comportamiento de los empresarios frente a los acuerdos de integración, hace parte de un estudio de la planeación en las organizaciones, que complementa el escrito Explorando los métodos de planeación en las organizaciones, publicado en la revista Questionar volumen No. 4. Su objetivo es reflexionar en el concepto de planeación a través de los modelos que han surgido como herramienta teórica que fundamentan su ejercicio en procura de mayor competitividad. Metodológicamente, el trabajo se basa en una exhaustiva revisión documental y aproximación epistemológica al tema. El resultado muestra que el concepto de planeación es dinámico, que prevé la incertidumbre y es valioso para afrontar la competitividad que generan los nuevos mercados que trae la globalización.

Palabras clave: planeación, modelo, organizaciones, globalización, competitividad.

\section{Abstract}

This article arises within the framework of the research project about employers' behavior towards integration agreements; it is part of a study of planning in organizations; it complements the paper Exploring methods of planning in organizations, published in the Questionar magazine volume No. 4. Its objective is to reflect on the concept of planning through the models that have emerged as a theoretical tool that bases their exercise in pursuit of higher competitiveness. Methodologically, the work is based on an exhaustive documentary review and epistemological approach to the subject. The result shows that the concept of planning is dynamic, to foresee uncertainty and is valuable to face the competitiveness generated by the new markets that globalization brings.

Keywords: planning, model, organizations, globalization, competitiveness.

\footnotetext{
* Economista, magíster en Gestión Pública, candidato a doctor en Administración (Universidad de Celaya, México). Grupo de Investigación en Gestión y Competitividad de las Organizaciones, Fundación Universidad de América, Bogotá, Colombia. ORCID: http://orcid.org/0000-0003-3797-0249. desiderio.lopez@ investigadores.uamerica.edu.co ** Ingeniera Química, Especialista en Gerencia de Empresas. Fundación Universidad de América, Bogotá, Colombia.yeimi.0709@ hotmail.com
} 


\section{INTRODUCCIÓN}

El auge de la globalización conlleva que las organizaciones deban recorrer caminos que conduzcan a ganar más competitividad, vías que en algunas oportunidades los líderes las llaman estrategias; en otras situaciones, toma de decisiones, y en otras, proyección (Palacio, 2010). Indiferente al nombre que se emplee, el ejercicio de vislumbrar el camino supone recorrer una empresa empleando sus recursos de una manera eficiente, en procura de hacer presencia en la mayor cantidad de mercados posibles, lo que se denomina planeación (George y Alvarez, 2005). Tanto el diseño de estrategias, como la toma de decisiones y la misma planeación, implican un proceso de análisis sistemático de la realidad presente y un cálculo de las posibles evoluciones de esa realidad, que usualmente se conoce como planeación estratégica (Aguilar, 2006). Dadas las diversas denominaciones o acepciones sobre planeación aquí referidas, en este escrito no se profundizará sobre ellas, sino se ocupará de ver los diferentes modelos de planeación.

Esas concepciones sobre planeación lo único que han generado es una confusión entre planeación y estrategia, que poco le han aportado a la competitividad de las organizaciones, a lo cual se le suma la disputa conceptual sobre el término planeación estratégica, que en palabras de Aguilar (2006), a esa combinación de vocablos "no se le han revisado sus resultados" (p. 247). Por esta razón, el objetivo de este escrito es reflexionar sobre el concepto del término planeación reflejado en sus diferentes modelos, como una herramienta teórica que fundamenta el actuar de la organización en busca de mayor competitividad. Lo aquí planteado se complementa con lo descrito en el artícu$10^{1}$, mencionado anteriormente; los dos trabajos permiten ver en forma holística el papel que juega la planeación en las organizaciones, para lograr una incursión consciente en nuevos mercados o incrementar su presencia en ellos.

Es clave que las organizaciones tengan la capacidad para construir modelos de planeación que sean adaptables, que sean flexibles y que sean potentes, aún en las circunstancias más desfavorables de información, de relaciones adversas o incluso en un mundo cambiante y vertiginoso. Aquí y ahora es cuando más se requiere de la planeación para generar una mayor competitividad y participación en los mercados internacionales.

El documento se inicia con la descripción de la metodología empleada en la búsqueda de los escritos aquí referidos, continúa con una aproximación a la fundamentación de la planeación, donde se exploran algunos conceptos que han manifestado los expertos del tema, lo cual permite precisar el alcance de la acepción, su evolución y su ámbito. Enseguida se hace referencia a los modelos de planeación, bajo la premisa que la planeación es una herramienta del actuar de las organizaciones y a éstas las estudian las ciencias sociales, lo que conlleva generar una mirada más allá de las teorías neoclásicas que han acogido los modelos matemáticos como centro de estudio, hasta alcanzar campos que se centran en el análisis de variables y del pensamiento sistémico, como los modelos cualitativos y los analíticos. Al final se hace una sección a manera de conclusión, dando cuenta de la dinámica del concepto de planeación, que como herramienta teórica fundamenta, mediante modelos, el proceso de decisiones que prevé la incertidumbre y es valiosa para afrontar la competitividad que generan los nuevos mercados que trae la globalización.

\section{Metodología}

El concepto de planeación y sus diferentes modelos que se aplican en las organizaciones han sido abordados desde diferentes campos de estudio y han variado según la época, por ello el estudio que se realizó es de carácter reflexivo y no se enmarca dentro de ninguna escuela de pensamiento or-

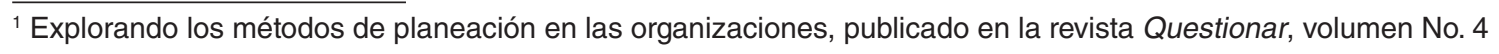


ganizacional, aquí se considera que el vocablo planeación ha tenido varias lecturas, sus promotores la han sobrevalorado y sus detractores se refieren a ella en términos peyorativos.

La investigación se desarrolló con base en una revisión de la literatura sobre el término planeación, partiendo de la síntesis que publicó Aguilar (2006) sobre el surgimiento del pensamiento estratégico en la década del sesenta de la centuria pasada en la Escuela de Negocios de Harvard, luego el trabajo se concentró en artículos recuperados mediante el empleo de bases de datos suscritas por la Universidad de América como Emerald, Ebsco, Science Direct y Springer Link, y el servicio de Celsius, lo cual permitió revisar y organizar los escritos de acuerdo a los modelos que más se emplean en las organizaciones en procura de mejorar su competitividad.

\section{Fundamentación de la planeación}

La planeación hace parte de los objetos de estudio de las organizaciones, y éstas se clasifican como un tema de las ciencias sociales; Waldo (2006) al respecto dice: "La planificación son los medios por los cuales la ciencia aplicada a las cuestiones humanas puede facultarle al hombre encarnar sus propósitos" (p. 24). En otras palabras, el estudio de la planeación se centra en el análisis de la comprensión, del como el ser humano relaciona los medios con los fines para alcanzar los objetivos de su organización.

El carácter humano que esta implícito en el ejercicio de la planeación de las organizaciones provoca diversidad de miradas sobre la forma como se diseña e implementa esa herramienta teórica, o sea que, cada proceso de planeación tiene sus particularidades en concordancia con los objetivos que se persiguen y de las decisiones que se adopten al interior de la organización.

Este proceso en general requiere de una argumentación que se sustente en una teoría ante la ausencia de una teoría general de planeación, es necesario recurrir a modelos sobre el término de "teoría" en la línea de Maxim, (2002) que lo define como: "conjuntos de enunciados verbales que sintetizan el comportamiento de sistemas empíricos o brindan una explicación suficiente que permite comprender por qué tales sistemas se comportan como lo hacen [...]. Pero en las ciencias sociales existen dificultades para cumplir con esa definición" (p. 30). Maxim insiste que cualquier comportamiento requiere de una explicación para comprender su actuar y que ello sólo se logra mediante una teoría, no obstante aclara que ante su ausencia "muchos científicos sociales han optado por el termino modelo para describir sus enunciados teóricos” (p. 31). Siguiendo este planteamiento, la planeación se fundamenta en los modelos que aquí se exponen. Antes de abordar los modelos de planeación, es importante profundizar en el alcance y madurez del término.

El concepto de planeación y su aplicación tiene tanto promotores como detractores. Después de la segunda guerra mundial surge el concepto de planificación para la reconstrucción de los países afetados, y en 1960 se adopta el concepto de planeacion en las organizaciones. Acerca de la gestación, maduración y crítica a la planeación, es interesante traer la síntesis que hace el profesor Aguilar (2006):

Ubicar el renacimiento del pensamiento estratégico en los años sesenta es justificado, porque en esa década se publican las tres obras que, además de pioneras, mantienen su influencia hasta la fecha en los desarrollos teóricos y en los ejercicios profesionales de dirección y/o planeación estratégica empresarial. Estas tres obras son: Estrategia y estructura (1962), el libro histórico-teórico de Alfred D. Chandler; el libro inaugural Estrategia corporativa (1965) de Igor Ansoff, y el influyente libro de texto de la Escuela de Negocios de Harvard, La política de negocios: texto y casos (1965), editado conjuntamente por E. Learned, R. Christensen y Kenneth R. Andrews, a quien se atribuye el texto conceptual sobre el método de análisis y gestión estratégica y que publicó después otros libros refinando el enfoque. Estas obras fundadoras fueron posteriormente continuadas y simplificadas por los trabajos de índole práctica de George A. Steiner, recapitulados en Planeación estratégica (1979), y llegaron 
a su rebase y replanteamiento en los tres libros capitales de Michael E. Porter, Estrategia competitiva (1980), Ventaja competitiva (1985), La ventaja competitiva de las naciones (1990), cuya influencia académica y empresarial ha sido dominante .en los últimos años. Otro autor fundamental, crítico de la planeación estratégica convencional pero con aportes sugerentes, es H. Mintzberg, con su trilogía El proceso estratégico (1991), The Rise and Fall ofStrategic Planning (1994) y Safan a la estrategia (1998). Y cierra el grupo de las obras merecedoras de atención el libro de G. Hamel y C. K. Prahalad, Competir para el futuro (1994), que revierte posiciones consagradas e introduce una perspectiva diferente (p. 246)

Entre los autores citados por Aguilar (2006), como pioneros de la planeación se observa que (Chandler, 2009), toma prestado el concepto de planificación que venían utilizando los organismos internacionales y lo adapta para las organizaciones con el término de planeación, asociándolo a una estrategia de largo plazo, así: "definir los objetivos y metas de largo plazo de una empresa y elegir los cursos de acción y asignar los recursos para poder realizar los objetivos y metas" (p. 447). Pero el gran salto lo hace la Escuela de Negocios de Harvard que con su método inductivo de estudios de caso, recoge experiencias de diferentes organizaciones y lanza el método SWOT, el cual se abordó en el artículo Explorando los métodos de planeación en las organizaciones, de López-Niño y Cárdenas, (2016) son las letras iniciales de las palabras en lengua inglesa: strengths, weakneses, opportunitis, theats, y que en lengua española se conocen como: debilidades, oportunidades, fortalezas y amenazas (DOFA). Método que fue profundizado por Kenneth R. Andrews, y que a pesar de las fuertes críticas todavía se emplea en los procesos de planeación en diversas organizaciones, en él se analiza la situación interna de la organización y su entorno, relacionando sus capacidades con las opciones universales.

Es inegable que desde las postrimerias del siglo pasado, se vive una época signada por la incertidumbre y lo impredecible lo que conlleva que el ejercicio de la planeación esté cuestionado, sin embargo Michael E. Porter, (2015) en la década del noventa de la centuria pasada, le ha lanzado a la planeación un salvavidas, por cuanto la mantiene como elemento subyacente en sus teorías, vinculando los beneficios de las empresas a la planeación estratégica, observa lo siguiente:

Toda empresa de una industria tiene una estrategia competitiva ya sea explícita o implícita. Pudo haberla desarrollado en forma explícita (en un proceso de planeación) o implícita (en las actividades de sus departamentos funcionales) [...\} El énfasis que está puesto en la planificación estratégica de las empresas actuales en Estados Unidos Y otros países refleja la convicción de que se obtienen importantes beneficios mediante la formulación explícita de la estrategia (p. xxi).

Pero esa visión de una planeación mecánica, ha sido criticada por Henry Mintzberg (1997), quien desde tiempo atrás ha venido afirmando que los planteamientos de Ansoff (1965) y los casos de estudio de Harvard (1965) se fundamentan en un racionalismo que conllevan a una prescripción en la formación de la estrategia, pero no crean la estrategia. Es importante tener en cuenta que Mintzberg insiste en que la planeación no solamente es racional, sino también intuitiva, lo que implica combinar diversas percepciones de la realidad, y que en este sentido se ha pronunciado el Foro Iberoamericano Sobre Estrategia de Comunicaciones (FISEC) mencionando que las organizaciones deben ser visionarias y estar alertas a todos los cambios, para desarrollar un pensamiento complejo, conjugando más variables:

Las organizaciones de hoy pueden ser definidas en su complejidad por los conceptos de las nuevas ciencias. Sus prioridades fundamentales reposan sobre la dinámica del cambio y las interfaces con el entorno exterior, más que sobre su estructura y sus recursos internos. Posee las características de un sistema abierto que procede de manera constante a su auto-organización y que es capaz de aprender 
a través de interacciones en el seno de su estructura o entre sus empleados y los actores exteriores (proveedores, clientes, comunidades políticas, sistemas ecológicos). (Perez y Massoni, 2009, p. 220)

Al percibir a las organizaciones como un sistema abierto donde interactúan múltiples actores tanto internos como externos conlleva que su planeación considere el futuro como algo desconocido. Ello implica la necesidad de profundizar en el estudio de la planeación desde la complejidad de hoy, hasta donde su fundamentación logre explicar, mediante modelos, el comportamiento de los empresarios y las decisiones que ellos tomen, considerando sus paradigmas y valores, en procura de mantener la competitividad de sus organizaciones (Dror, 2005).

\section{Modelos de planeación}

Como se ha mencionado la planeación es una herramienta teórica de la gestión en las organizaciones, y su campo de estudio hace parte de las ciencias sociales (Sanabria, Saavedra y Smida, 2014), su fundamentación se soporta en modelos, entendiendo como modelo "describir enunciados teoricos en intentos por construir teorias" (Maxim, 2002, p. 31). Existen diferentes clasificaciones de modelos, los parametros o criterios que establecen los expertos para hacer esas clasificaciones no son objeto de análisis en este artículo, pero por el carácter epistemologico del artículo es importante hacer una anotación sobre las diversas categorias de modelos que establecen algunos autores.

En primer lugar, según Lévi-Strauss (1987, citado por Ruiz y Padilla, 2012), en las ciencias sociales se emplean dos tipos de modelos: los modelos mecánicos y los modelos estadísticos. Los primeros quieren explicar los mecanismos, factores o procesos que producen determinado estado de cosas; en los segundos se inducen regularidades o correlaciones entre diversas series de fenómenos. En segundo lugar, según Vickers (2015), los modelos se clasifican en modelos de medición y modelos de trayectoria, el anaísis de datos se hace en una de las dos categorias, por cuanto algunos implican la construcción de medición y otros la determinación de las relaciones entre las diferentes construcciones. En tercer lugar, según Dror (2005), en las ciencias sociales se identifican tres tipos de modelos: cuantitativo, cualitativo y analítico. Para este escrito se opta por la clasificación de Dror, considerando su especialización en temas administrativos.

\section{Modelo cuantitativo}

El modelo cuantitativo expresa mediante una fórmula matemática la relación recíproca entre distintas variables (Dror,2005). Modelo que se abrió paso en la disciplina de la Administración, a finales del siglo XIX cuando los pioneros del campo de estudio estaban probando el valor de las técnicas científicas en el área de planificación de la producción, lo cual dio los primeros pasos para que surgiera la administración científica de Frederick W. Taylor, y posteriormente surgiera la escuela cuantitativa de la Administración (Chiavenato, 2015).

La planeación en el modelo cuantitativo se fundamenta en la toma de decisiones empresariales, la cual ha evolucionado conjuntamente con el resto de la teoría de las organizaciones a un punto donde puede expresarse profundamente en términos matemáticos. El uso del cálculo permiten la expresión concisa de las relaciones funcionales complejas y la rápida solución del problema que implica la distribución optima de recursos en procura de la compettividad de las organizaciones (Keat y Young, 2004).

Esa solución de problemas y distribución de recursos, implica la adopción de decisiones, y ello supone elegir entre una pluralidad de opciones, la disciplina que estudia dicho proceso es la teoría de la decisión. La elección de la decisión se realiza mediante la aplicación de un criterio fundamentado en la teoría matemática y de la probabilidad. En este sentido, el conjunto de estos criterios constituyen 
los procesos de decisión que aplicados a problemas de índole económico se denominan métodos de decisión empresarial que buscan su competitividad (Rabadán, Cid, y Leguey, 2014).

En síntesis el modelo matemático (Rabadán, Cid y Leguey, 2014) está conformado por los siguientes elementos: decisor, quien es una persona o una dependencia que trata de alcanzar unos objetivos dentro de una organización; alternativas o decisiones, consideradas como son las vías para alcanzar los objetivos, variables que el decisor controla; estados de la naturaleza, son los parámetros que definen una situación de incertidumbre, variables que están por fuera del control del decisor; consecuencia o resultados, paso siguiente a la elección de una alternativa "puede considerarse una aplicación del producto cartesiano de las alternativas y estados de la naturaleza en los números reales" (p.2); información, es el conjunto de elementos relacionados con el estado de la naturaleza. Estos elementos conforman la fundamentación del modelo cuantitativo de la planeación en las organizaciones, construyen una herramienta teórica que en forma simple se puede representar así:

Alternativas o decisiones a

Estados de la naturaleza $\theta$

Conjunto de resultados $\mathrm{R}$

$R_{i j}=f\left(a_{i}, \theta_{j}\right)$

El modelo matemático resuelve principalmente la pregunta sobre que resultados se obtendrán a partir de una decisión que se tome, de acuerdo a las relaciones que generan las variables que controla el decisor y las variables que no controla, en concordancia con los objetivos que se ha fijado, y cómo aumentar la competitividad en los mercados globalizados. Claro está que el modelo cuantitativo resulta corto, cuando se presentan altos índices de incertidumbre y complejidad, como la época actual, dando cabida a otros modelos.

\section{Modelo cualitativo}

El modelo cualitativo determina las relaciones reciprocas de distintas variables, sin el uso de formulas matemáticas o clasificaciones cuantitativas (Dror, 2005). Al respecto, Aguilar (2006) afirma que:

La planeación es una definición clara de la relación que la organización establece entre medios-finés, causas-efectos, acciones-resultados. Es una cadena de medios-finés, causas-efectos, cuyos eslabones van precisados, pues hay metas intermedias cuya realización está en función de realizar objetivos mayores que, una vez realizados, son realidades que sirven a su vez de recursos-medios-condicionescausas para efectuar los objetivos finales. En este sentido, un plan es la expresión de "juicio A valor" sobre la ocurrencia de ciertos hechos — su deseabilidad, preferibilidad, imperatividad-y “juicios de hecho", juicios causales sobre la manera de producirlos, hacer que ocurran. (p. 235)

Además refleja las creencias y valoraciones de una organización, los valores que profesa y con los que está comprometida y toman forma en determinadas situaciones reales presentes y futuras. Plasma asimismo las creencias causales de la organización los procesos de acción considerados eficaces y/o eficientes para realizar la situación deseada. En este sentido un plan supone, refrenda o genera el sistema de valores y el sistema causal de una organización, la planeación es un ejercicio que combina valoración + prospección + estrategia, es decir: juicios de valor (futuros deseados) + juicios de hecho (futuros realizables, factibles) + agenda estratégica (futuros ganadores) (Aguilar, 2006). Los juicios como característica cualitativa le da la esencia relacional al modelo. Estos componentes soportan la fundamentación del modelo cualitativo de la planeación en las organizaciones, constituyéndose en una herramienta teórica en procura de fortalecer la competitividad de las organizaciones en el mundo globalizado imperante. 
Este modelo, ofrece una alternativa distinta a los principios idealizados de racionalidad, que en realidad hacen juicios intuitivos y compromisos, lo que parece ser la característica fundamental de su práctica. Es decir, ver desde una nueva perspectiva lo que se viene haciendo, construyendo relaciones inéditas. Por su carácter cualitativo, se confecciona a partir y desde los sentires de los participantes. La diferencia con los otros dos modelos que aquí se exponen es su ruptura con la racionalidad (Friend y Hicking, 2002), sin perder de vista que el objetivo de una organización es ganar mas mercados o fortalecerse en ellos, es decir, mejorar su competitividad.

Este modelo resuelve principalmente la pregunta sobre qué objetivos se fijan, considerando el sistema de valores de la organización y la combinación de valoración, prospección y estrategia. Ello permite que la tarea de planificar sea vista como un arte, lleno de sutilezas y desafíos, a través del cual la organización puede desarrollar sus proyectos de acuerdo a sus capacidades, de manera creativa para captar complejidades e incertidumbres de esta época, resultando un modelo con muchas potencialidades de cara a los nuevos retos de la globalización y la competitividad.

\section{Modelo analítico}

El modelo analítico se desarrolla a partir de un diagnostico de variables principales, su conceptualización y análisis sistemático (Dror, 2005). Desarrolla un proceso sistémico de toma de decisiones en busca de resultados que resuelva problemas (van Gigh, 2009), como la competitividad de las organizaciones. El modelo comprende los siguientes elementos: es un proceso, que conlleva la existencia de personas y recursos. Es la preparación de un conjunto de decisiones, que implica un sistema donde las decisiones son interdependientes, relacionadas unas con otras en miras de alcanzar unos objetivos. Se materializa en la actividad, no se queda en la reflexión, busca resultados distintos a los que se podrían conseguir sin ella. Una de las características básicas de la planeación es el tiempo futuro. Investigación, reflexión, y pensamiento sistémico y sistemático, la planeación es racional, no se basa en intuición e impulsos momentáneos (Dror, 2005).

Esos elementos se soportan desde cuatro perspectivas: a) El entorno, las condiciones externas; b) lo económico, la razón de ser de la organización; c) el plan, la concreción de lo investigado; d) las capacidades de la organización y de las personas que la integran (Dror, 2005). Tanto los elementos como las perspectivas conforman la fundamentación del modelo analítico de la planeación en las organizaciones, construyen una herramienta teórica que se describe de la siguiente forma:

Con relación al entorno. Por su esencia analítica, el modelo se apropia de la dinámica del entorno en la identificación de sus variables, considerando su naturaleza de variables independientes que pueden abrir o cerrar el camino en el logro de los objetivos. Lo cual significa reconocer que hay agentes, pautas de acción y eventos en el medio ambiente que son imprevisibles, incontrolables, inmanejables (Chandler, 2009). Aquí radica la diferencia frente a los dos modelos ya vistos, el cuantitativo y el cualitativo, por cuanto se considera la turbulencia que genera la globalización, pero no se pierde de vista que lo que se busca es la competitividad de las organizaciones considerando ese entorno.

Con relación a lo económico. Es un ejercicio analítico que permite a la organización su posicionamiento sobre los competidores, ya sea para beneficio de sí misma y/o para beneficio de los destinatarios de sus servicios, incluso para beneficio de la comunidad social (Perez y Massoni, 2009). Igualmente permite enfocarse hacia aquellos objetivos que aseguran que la organización en el futuro tenga éxito y liderazgo, que supere sus atrasos y enfrente retos como los de los nuevos competidores y/o los nuevos mercados que se derivan de los acuerdos de integración económica (Palacio, 2010). Consiste en ser capaz de producir un bien o prestar un servicio con un gradó o magnitud de valor superior al de los bienes y servicios que proveen otros agentes presentes en el mismo sector de actividad. (Porter, 2015) 
Respecto al plan. Es un documento de trabajo, que permite orientar a las organizaciones hacia una mayor competitividad, es una carta de navegación para un periodo definido. El formularlo no es suficiente, se requiere su implantación, mediante planes operativos, identificando responsables de la ejecución de los proyectos, con la elaboración de cronogramas de trabajo que identifiquen claramente las responsabilidades en el tiempo y con la asignación de los recursos requeridos (Friend y Hicking, 2002).

Frente a las capacidades. El logro del objetivo de aumentar la competitividad depende de las capacidades con que cuente la organización, sus principios y valores, lo mismo que su tecnologia tanto en los procesos operativos como administrativos, la organización en que se soporte la producción y el servicio que se suministra, las competencias del personal y la infraestructura (Keat y Young, 2004).

El modelo analítico resuelve principalmente la pregunta sobre qué decisiones se deben tomar en concordancia con el entorno fundamental en la realidad, es decir, revela sus reales componentes y sus relaciones en términos de problemas y potencialidades y los relaciona con los objetivos de la organización, lo cual resulta altamente favorable para fortalecer la competitividad de las organizaciones que aspiran a participar en el campo que crea los cuerdos de integración económica. Uno de los modelos analíticos que más acogida ha tenido entre las empresas que buscan incursionar en los mercados europeos es el European Foundation for Quality Managament (EFQM).

Es importante abordar el modelo EFQM, dada su relación con el objetivo de este escrito, fue implementado en el año 1988 por catorce empresas europeas, con el fin de aumentar su competitividad (Álvarez y Caballero 2013). La estructura del modelo contiene una retroalimentación basada en el desarrollo de facilitadores y resultados para llegar a la comprensión, creatividad e innovación de la planeación. "Este proceso de retroalimentación tiene una importancia fundamental, tanto para definir y planificar la formación especializada continua como las políticas de incentivos hacia el capital intelectual de la organización, al estimular ambas la mejora continua dentro de la empresa" (Álvarez y Caballero, 2013, p. 39)

Para comprender la metodología del EFQM, Membrado (2013), la expone en un esquema (figura 1) allí se identifican los criterios facilitadores, resultados y la retroalimentación (innovación $\mathrm{y}$ aprendizaje).

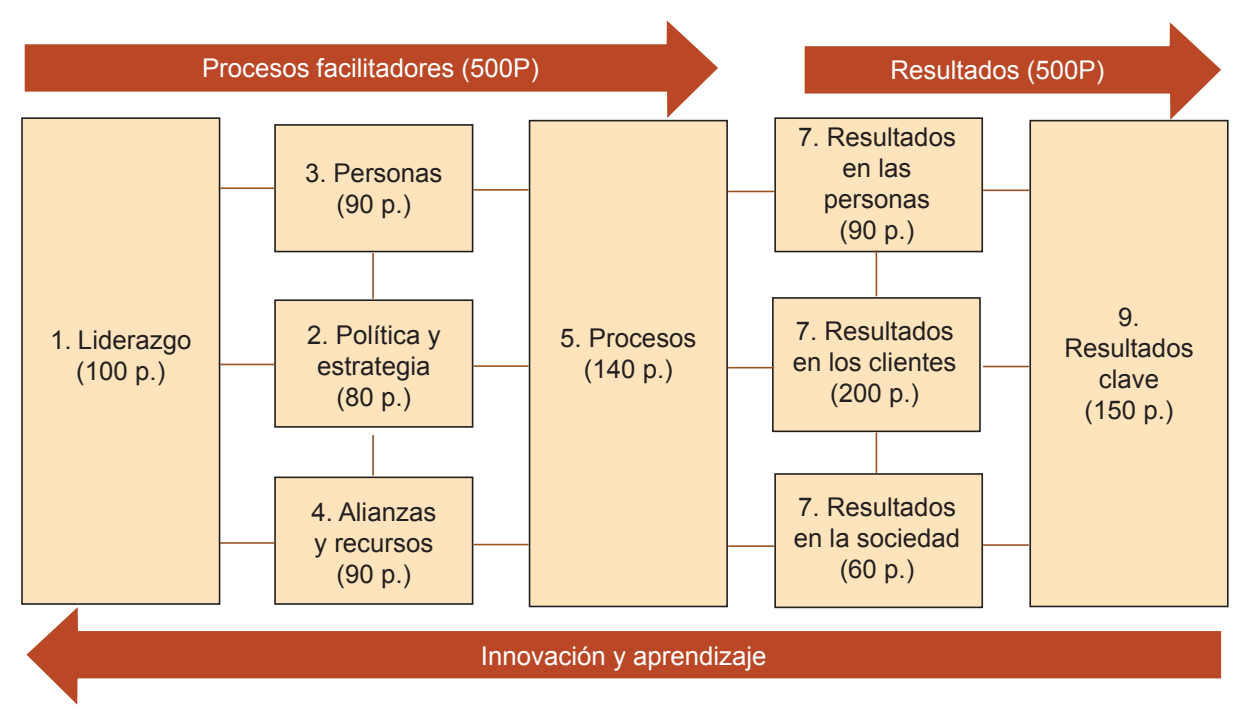

Figura 1. Modelo de excelencia EFQM y sus nueve criterios.

Fuente: Extraído y modificado por los autores de Membrado Martínez (2013). 
El éxito del modelo de excelencia EFQM radica en el cumplimiento de la metodología y el buen uso de los criterios (facilitadores y resultados), y subcriterios del método para obtener una mejor retroalimentación. "Del mismo modo, la Fundación Europea para la Gestión de Calidad afirma, en su página web que "la EFQM Modelo de Excelencia está siendo implementado por más de 30.000 industrias en el mundo ", pero esta industria da esa información sin ningún tipo de referencia a la fuente de los datos.” (Khaledi, 2015, pág. 16).

De todas maneras, varios expertos: Jiménez y Martínez (2015), Dutt, Biswas, Arora y Kar (2012), Ruiz y Fernández (2005), Ugalde (2009), Steed, Maslow y Mazaletskaya (2005) han analizado el modelo y mediante evidencia empírica han observado que el EFQM es un modelo bastante completo en su metodología, que ofrece beneficios para la competitividad de las organizaciones haciendo resaltar los conceptos fundamentales como parte clave de la excelencia basándose en una retroalimentación valedera que ha logrado éxito en su uso y aplicaciones.

\section{CONCLUSIONES}

La planeación es una herramienta teórica de las organizaciones, mediante modelos, permite construir caminos que les genera mayor competitividad, optimiza sus recursos y le da sentido a la toma de decisiones. La planeación se fundamenta en varios modelos como el matemático (cuantitativo), el cualitativo y el analítico; cada uno tiene identificado sus elementos y las relaciones entre ellos, de una manera dinámica, y considerando los distintos escenarios que puedan presentarse por los constantes cambios y avances no solo en ciencia y tecnología, sino por la galopante globalización y complejidad. Esa adaptación a las situaciones cambiantes garantiza que el concepto de planeación no quede obsoleto.

Tanto la globalización como la complejidad conllevan una nueva mirada sobre la planeación, donde su objeto no se limita a la elaboración de un plan, sino a fundamentar un proceso dinámico y una respuesta a la incertidumbre, como los retos que traen nuevos mercados dentro del marco de los acuerdos de integración económica que se vienen pactando en todo el orbe.

A lo largo del documento se dio cuenta que existen varias herramientas teóricas o modelos, para planificar en procura que las organizaciones afronten el reto de la globalización. Una de ellas, el modelo cuantitativo, se sustenta en la programación matemática, la cual permite llevar a cabo una búsqueda sistemática de soluciones dentro de un campo complejo y multidimensional, pero ese modelo resulta inapropiado cuando se trata de afrontar cambios demasiados rápidos, como los que suceden en esta época, de ahí la importancia de valorar una nueva mirada sobre la planeación. Una planeación que incorpore componentes signados por la complejidad, busque que lo deseable se haga factible y acepte que es prácticamente imposible prever con precisión los futuros que son discontinuos con el pasado, que su éxito depende de visualizar el futuro a partir de la comprensión del hoy, de tal forma que se logre minimizar los riesgos y aprovechar las oportunidades en procura de aumentar la competitividad de las organizaciones.

Para una mayor comprensión del concepto de planeación, sus autores muy respetuosamente sugieren complementar su lectura con el escrito realizado por ellos mismos, intitulado "Explorando los métodos de planeación en las organizaciones".

\section{REFERENICAS}

Aguilar, L. (2006). Gobernanza y Gestión Pública. México: Fondo de Cultura Económica.

Álvarez, J. y Caballero, B. (2013). Modelo EFQM y creación de valor: una aproximación teórica1. The Anábuac Journal: Business and Economics, 13(1), 31-54. 
DESIDERIO LÓPEZ NIÑO, YEIMI JIMENA CÁRDENAS SANTAMARÍA

Chandler, A. (2009). Scale and scope: The dynamics of industrial capitalism. Cambridge: Harvard University Press.

Chiavenato, I. (2015). Introducción a la Teoria General de la Administración (3a Ed.). México: McGraw Hill.

Dror, Y. (2005). Enfrentando el futuro. México: Fondo de Cultura Económica.

Dutt, M., Biswas, D., Arora, P., y Kar, N. (2012). Using the EFQM Model Effectively. Journal for Quality E Participation, 35(1), 11-17.

Friend, J., y Hicking, A. (2002). Planificando bajo presión. Caracas: Ministerio de Defensa, Venezuela.

George, C., y Alvarez, L. (2005). Historia del pensamiento administrativo. México: Pearson.

Gómez, J., Martínez Costa, M. y Martínez Lorente, Á. (2015). EFQM Excellence Model and TQM: an empirical comparison. Total Quality Management E Business Excellence, 28(1-2), 88-103

Keat, P., y Young, P. (2004). Economia de Empresa (4a Ed.). México: Pearson.

Khaledi, I. (2015). Investigation Various Effects of Using EFQM Model on Industries' performance. Researcher, 7(7), 15-18.

López-Niño, D.. y Cárdenas, Y. (2016). Explorando los métodos de Planeación en las Organizaciones. Questionar, 4(1), 17-30.

Maxim, P. (2002). Métodos cuantitativos aplicados a las ciencias sociales. México: Oxford University press.

Membrado, J. (2013). Metodologias avanzadas para la planificación y mejora. España: Ediciones Díaz de Santos.

Mintzberg, H. (1997). El proceso estratégico: conceptos, contextos y casos. (3a Ed.). México: Pearson.

Palacio, I. (2010). Colombia frente a la globalización. Bogotá: Universidad del Rosario.

Perez, R., y Massoni, S. (2009). Hacia una Teoria General de la Estrategia. Barcelona: Ariel

Porter, M. (2015a). Estrategia competitiva (2a Ed.). México: Grupo Editorial Patria.

Porter, M. (2015b). Ventaja Competitiva (2a Ed.). México: Grupo Editorial Patria.

Rabadán, A., Cid, A., y Leguey, S. (2014). Métodos de decision empresarial. Madrid: Delta Publicaciones.

Ruiz-Carrillo, J., y Fernández-Ortiz, R. (2005). Theoretical foundation of the EFQM model: the resource-based view. Total Quality Management \& Business Excellence, 16(1), 31-55.

Ruiz, C., y Padilla, L. (2012). Los modelos matemáticos en las ciencias sociales. Pensar. Epistemología y Ciencias Sociales, 7, 115-126.

Sanabria, M., Saavedra, J., y Smida, A. (2014). Los estudios organizacionales (1ª Ed.). Bogotá: Universidad del Rosario.

Steed, C., Maslow, D., y Mazaletskaya, A. (2005). The EFQM Excellence Model for Deploying Quality Management: A British-Russian Journey. Higher Education in Europe, 30, 307-319. 
UNA APROXIMACIÓN TEÓRICA A LA PLANEACIÓN EN LAS ORGNIZACIONES

Ugalde, M. (2009). E1 Modelo EFQM aliado en la adversidad. Qualitas hodie: excelencia, desarrollo sostenible e innovación, 143, 14-15.

Van Gigh, J. (20 09). Teoria general de sistemas aplicada. México: Trillas.

Vickers, R. (2015). Statistics and the art of model construction. The Science and Simulation of Human Performance, 5, 393-467.

Waldo, D. (2006). The administrative state: A study of the political theory of American public administration. Chicago: Transaction Publishers. 